\title{
Downregulation of autophagy by Bcl-2 promotes MCF7 breast cancer cell growth independent of its inhibition of apoptosis
}

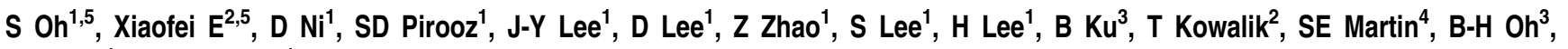 \\ JU Jung ${ }^{1}$ and C Liang ${ }^{\star 1}$
}

The anti-apoptotic Bcl-2 protein, which confers oncogenic transformation and drug resistance in most human cancers, including breast cancer, has recently been shown to effectively counteract autophagy by directly targeting Beclin1, an essential autophagy mediator and tumor suppressor. However, it remains unknown whether autophagy inhibition contributes to Bcl-2-mediated oncogenesis. Here, by using a loss-of-function mutagenesis study, we show that Bcl-2-mediated antagonism of autophagy has a critical role in enhancing the tumorigenic properties of MCF7 breast cancer cells independent of its anti-apoptosis activity. A Bcl-2 mutant defective in apoptosis inhibition but competent for autophagy suppression promotes MCF7 breast cancer cell growth in vitro and in vivo as efficiently as wild-type Bcl-2. The growth-promoting activity of this $\mathrm{Bcl}-2$ mutant is strongly correlated with its suppression of Beclin1-dependent autophagy, leading to sustained 62 expression and increased DNA damage in xenograft tumors, which may directly contribute to tumorigenesis. Thus, the anti-autophagic property of Bcl-2 is a key feature of Bcl-2mediated oncogenesis and may in some contexts, serve as an attractive target for breast and other cancer therapies.

Cell Death and Differentiation (2011) 18, 452-464; doi:10.1038/cdd.2010.116; published online 1 October 2010

Breast cancer is the most common malignancy and the secondleading cause of cancer-related deaths in women in the United States. Despite extensive studies regarding breast cancer, much is still unknown about the biological mechanism by which a normal cell turns cancerous. One critical factor involved in this process is $\mathrm{Bcl}-2$, which is highly expressed in $40-80 \%$ of breast cancer patients and is also common in other tumors. ${ }^{1}$ The conventional view of $\mathrm{Bcl}-2$ as a proto-oncogene focused on its ability to prevent apoptosis that contributes to the elimination of cancerous cells. ${ }^{2}$ Apoptosis is tightly regulated by the interplay of the Bcl-2 family proteins characterized by the presence of up to four conserved 'Bcl-2 homology' $(\mathrm{BH})$ domains. ${ }^{2}$ Members of the Bcl-2 family are classified as either anti-apoptotic (e.g. Bcl-2) or pro-apoptotic (e.g. Bax and Bak, Bid). ${ }^{3}$ The antiapoptotic Bcl-2 proteins block apoptosis by sequestering pro-apoptotic ones from inducing apoptosis. Of particular relevance, the molecular surfaces of Bcl-2's possess a BH3binding groove, an extended hydrophobic cleft formed by the juxtaposition of the $\mathrm{BH} 1-3$ domains, which can accommodate the $\alpha$-helical BH3 domain of the proapoptotic Bcl-2 molecules. ${ }^{4,5}$ Thus, the interactions between anti- and pro-apoptotic Bcl-2 proteins regulate the balance of cells' life and death and determine the propensity of cells to succumb to apoptosis.
Although intense efforts have focused on the anti-apoptotic role of $\mathrm{Bcl}-2$, recent evidence; however, uncovered a critical role of $\mathrm{Bcl}-2$ to concomitantly prevent autophagy. ${ }^{6,7}$ In contrast to the 'self-destruct' apoptosis, autophagy ('self-eating') involves the lysosome-dependent bulk degradation of cytoplasmic components, through a centrally important double-membrane-bound vesicle, the autophagosome. Formed within the cell, autophagosomes serve to surround, sequester, and finally, seal off extraneous cellular components from the rest of the inside of the cell. Autophagosomes subsequently fuse with lysosomes, having their sequestered contents degraded and the resulting macromolecules recycled. ${ }^{8,9}$ Although initially recognized as a response to nutrient deprivation, autophagy is now implicated as being essential to a variety of cellular processes including stress adaptation, development, immunity, and protection against neurodegeneration and cancer. ${ }^{8,9}$

$\mathrm{Bcl}-2$ suppresses autophagy by directly targeting Beclin1, a component of the class III PI3K complex involved in the autophagosome formation. ${ }^{6,10}$ An allelic loss of beclin1 impairs autophagy and renders mammary cells tumor-prone, suggesting that defects in Beclin1-mediated autophagy are essential for malignant transformation. ${ }^{11,12}$ Although the

\footnotetext{
${ }^{1}$ Department of Molecular Microbiology and Immunology, University of Southern California, Los Angeles, CA, USA; ${ }^{2}$ Department of Molecular Genetics and Microbiology, University of Massachusetts Medical School, Worcester, MA, USA; ${ }^{3}$ Department of Biological Sciences, Korea Advanced Institute of Science and Technology, Daejeon, Republic of Korea and ${ }^{4}$ Department of Pathology, University of Southern California, Los Angeles, CA, USA

${ }^{*}$ Corresponding author: C Liang, Department of Molecular Microbiology and Immunology, University of Southern California, Room 5517, MC NRT 9605, 1450 Biggy Street, Los Angeles, CA 90033, USA. Tel: 323442 7840; Fax: 323442 7868; E-mail: chengyu.liang @usc.edu

${ }^{5}$ These authors contributed equally to this work.

Keywords: Bcl-2; autophagy; apoptosis; MCF7; tumorigenesis

Abbreviations: Bcl-2, B-cell lymphoma 2; BH, Bcl-2 homology; MMP, mitochondrial membrane permeabilization; PI3K, phosphatidylinositol 3-kinase; $\gamma$-HV68, $\gamma$-herpesvirus 68; WT, wild-type; TM, transmembrane; Co-IP, co-immunoprecipitation; STS, staurosporine; TNF $\alpha$, tumor necrosis factor-alpha; p62, SQSTM1/ sequestosome 1; TUNEL, terminal deoxynucleotidyl transferase dUTP nick end labeling; UPR, unfolded protein response; IHC, immunohistochemistry; PARP, poly(ADP-ribose) polymerase; mTOR, mammalian target of rapamycin; FLIP, FLICE-like inhibitory protein; TSC1/TSC2, tuberous sclerosis 1 and 2; H\&E, hematoxylin/ eosin; HPF, high-power fields

Received 23.12.09; revised 17.8.10; accepted 17.8.10; Edited by E Baehrecke; published online 01.10.10
} 
precise mechanism governing Beclin1-mediated tumor suppression is still elusive, recent studies have demonstrated that autophagy defects in tumors cause accumulation of unwanted protein aggregates such as the p62 and ER chaperons, oxidative stress, and genome damage, all of which concomitantly fuel tumor growth. ${ }^{13,14}$ It is within this context we postulate that the inhibition of the tumor suppressor Beclin1 by $\mathrm{Bcl}-2$ may contribute to the oncogenic potential of $\mathrm{Bcl}-2$. In support of this view, it has been shown that when Beclin1 function is left unchecked by $\mathrm{Bcl}-2$, excessive levels of autophagy induce cell death in breast cancer cells. ${ }^{6}$ Moreover, knocking down $b c l-2$ results in autophagic rather than apoptotic cell death in MCF7 cells, suggesting that an alternative and/or additional mechanism involving autophagy may have a role in $\mathrm{Bcl}-2$-mediated oncogenesis. ${ }^{15}$ Most notably, our recent study on a viral $\mathrm{Bcl}-2$ (vBcl-2) encoded by $\gamma$-herpesvirus 68 ( $\gamma$-HV68) clearly indicated that the inhibition of autophagy by the vBcl-2-Beclin1 interaction directly contributes to persistent infection, a prerequisite for the malignant transformation of infected cells. ${ }^{16}$ All these observations raise the strong possibility that autophagy is tumorsuppressive and that Bcl-2-mediated blockade of autophagy may contribute to $\mathrm{Bcl}-2$ oncogenicity.

Structural analysis revealed that the interaction of Beclin1 with $\mathrm{Bcl}-2$ is reminiscent of that of pro-apoptotic $\mathrm{Bcl}-2$ proteins, in that Beclin1 has a putative $\alpha$-helical $\mathrm{BH} 3$ domain that allows it to dock into the hydrophobic groove of $\mathrm{Bcl}-2 .^{7,17}$ As the $\mathrm{BH} 3$-binding groove of $\mathrm{Bcl}-2$ is engaged by both proapoptotic and pro-autophagic molecules, mutations in the groove that block Beclin1 binding also eliminate binding to pro-apoptotic Bcl-2's and vice versa thus far, further complicating efforts to genetically dissect the in vivo role of Bcl-2 in cancer. As such, the role of Bcl-2 antagonism of autophagy in tumor development remains ill defined and the underlying mechanism is unclear.

In this study, we first used loss-of-function mutagenesis to identify functional domains that can differentiate between Bcl-2's anti-autophagic binding and its anti-apoptotic interaction. Further, using an in vitro MCF7 breast cancer cell-culture model and an in vivo mouse xenograft model, we observed that a Bcl-2 mutant that no longer inhibits apoptosis but retains its anti-autophagy function promotes the tumorigenic properties of MCF7 cells to a level similar to wild-type (WT) Bcl-2. This effect was specifically due to the inhibition of autophagy and was dependent on having an intact Beclin1 binding. Our findings thus demonstrate an oncogenic role of Bcl-2mediated autophagy inhibition in breast cancer. Unlike what was previously thought, that anti-apoptosis features prominently in the functions of $\mathrm{Bcl}-2$ in vivo, our study suggests that the anti-autophagic property of $\mathrm{Bcl}-2$ may be, at least in some contexts, eminently exploited in human cancer, thereby making it an attractive target for cancer therapy.

\section{Results}

Bcl-2 interacts with Beclin1 independently of its interaction with pro-apoptotic $\mathbf{B c l}-2$ proteins. To probe the specific role of Bcl-2-mediated inhibition of autophagy in cancer, we first sought mutants able to differentiate the anti-autophagic function of $\mathrm{Bcl}-2$ from its anti-apoptotic activity. To this end, we conducted a loss-of-function mutagenesis by creating $\mathrm{Bcl}-2$ mutants containing deletions in its functionally conserved domain, including mutants deleted of the $\alpha 1$ helix (residues 1-22) at the $\mathrm{N}$-terminus $(\mathrm{Bcl}-2 \Delta \alpha 1)$, the $\mathrm{BH} 2$ domain $(\mathrm{Bcl}-2 \Delta \mathrm{BH} 2)$, and of a triple alanine substitution at the 'WGR' signature motif of Trp144, Gly145, and Arg146 in the $\mathrm{BH} 1$ domain (Bcl-2AAA) (Figure 1a). These mutants were then tested for their ability to associate with Beclin1 and Bax using co-immunoprecipitation (co-IP). When coexpressed in 293T cells, Flag-tagged Bax efficiently co-immunoprecipitated with WT $\mathrm{Bcl}-2$, whereas the $\triangle \mathrm{BH} 2$ and the AAA mutations abrogated Bax interaction (Figure 1b). Unlike the other three $\mathrm{BH}$ domains, the $\alpha 1$ helix of the $\mathrm{BH} 4$ domain does not participate in the formation of the hydrophobic central core. However, the deletion of the $\alpha 1$ helix renders $\mathrm{Bcl}-2$ unable to bind Bax (Figure 1b). Similar data were obtained probing for endogenous Bax interaction with these Bcl-2 mutants (Figure 1c). Likewise, the bacteria purified GST-fused Bak (referred to as GST-Bak $\Delta$ TM; the TM domain was removed to increase its solubility in Escherichia coli) readily pulled down WT, but not mutant Bcl-2's in 293T (Figure 1d). Collectively, these data indicate that the $\mathrm{N}$-terminal $\alpha 1$ helix, in addition to the $\mathrm{BH} 1$ and $\mathrm{BH} 2$ domains, is essential for Bcl-2's heterodimerization with Bax and Bak, consistent with previous reports. $^{18}$

To determine whether the different $\mathrm{Bcl}-2$ mutants preserve their binding with Beclin1, 293T cells were transfected with the WT or mutant Bcl-2 together with Beclin1-V5. We found that the $\triangle \mathrm{BH} 2$ and AAA mutant Bcl-2's that failed to bind Bax also failed to bind Beclin1 (Figure 1e). Interestingly, Bcl-2 $2 \alpha 1$ that lost its ability to interact with Bax and Bak retained efficient Beclin1 binding similar to that of WT (Figure 1e). Analogous results were obtained with endogenous Beclin1 (Figure 1f). These data thus indicate that $\mathrm{Bcl}-2$ forms a complex with Beclin1 independent of its interaction with the pro-apoptotic $\mathrm{Bcl}-2$ proteins, and that the $\alpha 1$-helical region outside the core seems to contribute mainly to its anti-apoptotic binding.

The $\alpha \mathbf{1}$ helix is required for the anti-apoptotic function of Bcl-2. To determine whether the Bcl-2 mutants were competent in apoptotic inhibition, we established stable cell lines expressing equivalent levels of WT or mutant Bcl-2 (Supplementary Figure S1a). As shown in Figure 2a, markedly increased cell death was observed with cells expressing vector or the Bcl-2 mutants as compared with that of WT. Apoptosis inhibition was further evaluated using propidium iodide staining to check for the sub-G1 accumulation of cells. Consistently, the Bcl-2 mutants unable to interact with Bax and Bak failed to suppress STS-induced apoptosis, whereas Bcl-2WT expression conferred over $90 \%$ protection against apoptosis (Figure 2b). In support of this, unlike WT, none of the Bcl-2 mutants could inhibit STS-elicited caspase-3 activation and cleavage as measured by flow cytometry (Figure 2c) and immunoblotting (Supplementary Figure S1b), respectively. Similar results were observed with other apoptotic stimuli including etoposide, $\mathrm{TNF} \alpha$ and cycloheximide (data not shown). These data indicate that deletion of the $\alpha 1$ helix or 
a
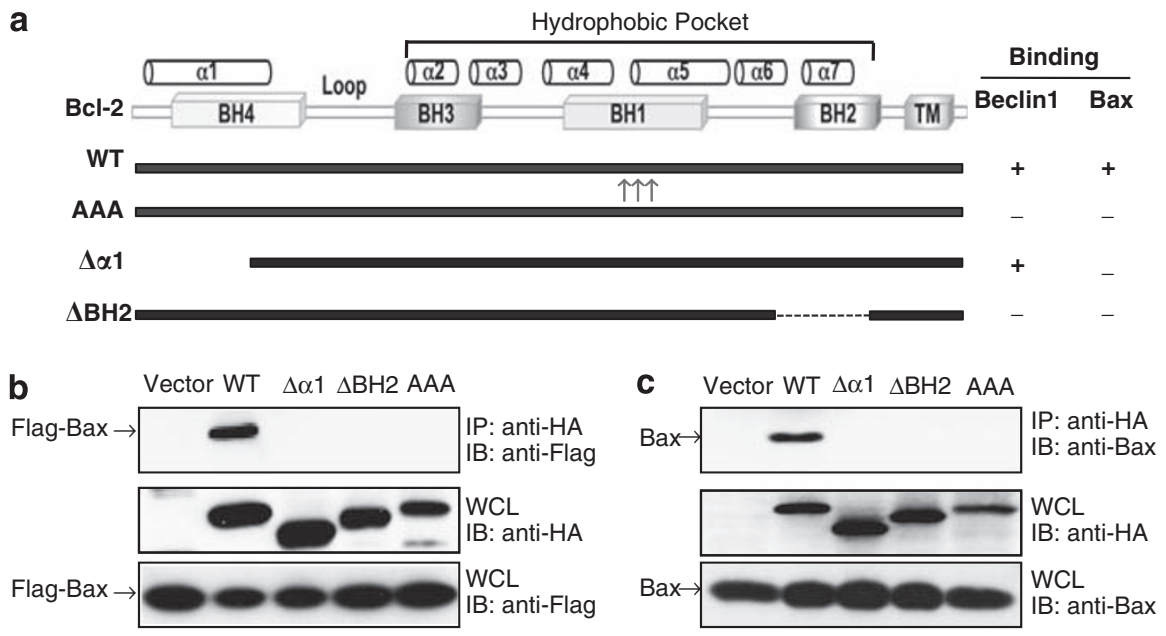

d
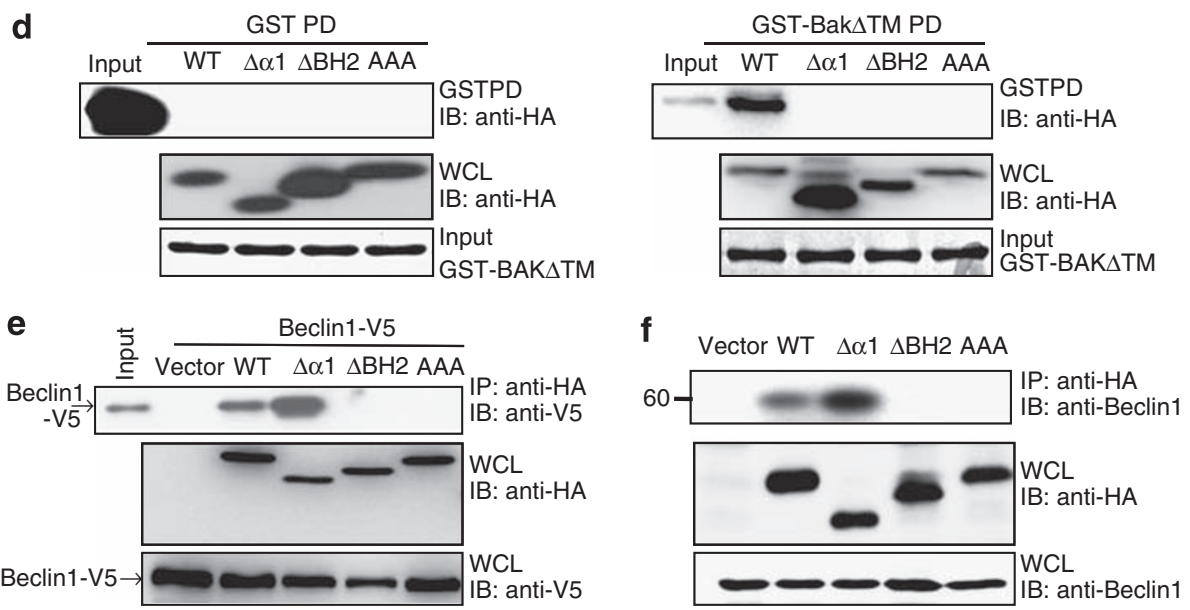

Figure 1 Anti-apoptotic and anti-autophagic interaction of WT and mutant Bcl-2 proteins. (a) Schematic representation of the wild type (WT) and mutant Bcl-2 constructs interacting with Beclin1 and/or Bax. The $\alpha$-helical structures of Bcl-2 is indicated at the top numbered according to previous publication. ${ }^{32}$ Colored boxes denote the BH 1-4 domains in Bcl-2; TM, transmembrane domain; '+ ' : positive interaction, '-': no interaction. Triple arrows denote the alanine substitutions at Trp144-Gly145-Arg146 within the BH1 domain of Bcl-2. (b and c) Co-immunoprecipitation (Co-IP) of WT and mutant Bcl-2 with Bax. 293T cells were transfected with the indicated Bcl-2 constructs, with (in b) or without (in c) Flag-Bax. Whole-cell lysates (WCLs) were used for immunoprecipitation (IP) with an anti-HA antibody, followed by immunoblotting (IB) with an anti-Flag (b) or a Bax (c) antibody. (d) Bcl-2 interaction with Bak. 293T cells were transfected with WT and mutant forms of Bcl-2 as indicated. At $48 \mathrm{~h}$ posttransfection, WCLs were mixed either with GST-Bak $\triangle T M$ fusion protein (right panel) or with GST alone (left panel) for an in vitro GST pulldown (GST PD) assays. GST fusion proteins used for the pulldown assay are indicated (bottom panel). $1 \%$ WCL was used as the input. Data are representative of at least three experiments yielding similar results. (e and f) Co-IP of WT or mutant Bcl-2 with Beclin1. 293T cells were transfected with the indicated constructs, followed by IP with an anti-HA antibody and IB with an anti-V5 (e) or an anti-Beclin1 (f) antibody. $1 \%$ whole-cell lysates (WCLs) was used as the input

any disturbance of the hydrophobic groove can severely impair the ability of Bcl-2 to suppress apoptosis. Thus, the $\alpha 1$ helix, which does not mediate Beclin1 interaction, is critical for the Bcl-2-mediated apoptosis antagonism, further supporting the existence of an anti-apoptosis-independent interaction between $\mathrm{Bcl}-2$ and Beclin1.

The anti-apoptosis defective $\mathrm{Bcl}-2 \Delta \alpha 1$ mutant inhibits Beclin1-dependent autophagy. As Bcl-2 lacking the $\alpha 1$ helix is unable to inhibit apoptosis, we sought to determine whether this region of $\mathrm{Bcl}-2$ is equally or differentially required for Beclin1-dependent autophagy. We initially used NIH3T3 cells stably expressing Bcl-2's as described above. Autophagy was evaluated using the fluorescent autophagosome marker, GFP-LC3, which redistributes from a diffuse cytosolic/nuclear staining to a cytoplasmic punctate pattern upon autophagy stimulation. ${ }^{19}$ Consistent with its Beclin1-binding ability, Bcl-2 $2 \alpha 1$ inhibits rapamycin- and starvation-induced GFP-LC3 puncta as effectively as WT. In contrast, the $\triangle \mathrm{BH} 2$ and $\mathrm{AAA}$ mutants showed almost no effect (Figure $3 a$ and Supplementary Figure S1c). To further validate this, we analyzed the lipidation of LC3 by immunoblotting. During autophagy, cytosolic LC3 (LC3-I) covalently conjugates to phosphatidylethanolamine to yield a lipidated form of LC3 and LC3-II, which displays a higher electrophoretic mobility and is indicative of autophagy. ${ }^{20}$ As seen in the GFP-LC3 puncta assay, the production of LC3-II was greatly reduced in the WT- and $\Delta \alpha 1$-expressing cells compared with that in the $\triangle \mathrm{BH} 2$ - or AAA-expressing cells (Figure $3 b$ ). To verify that the reduced levels of LC3-II 

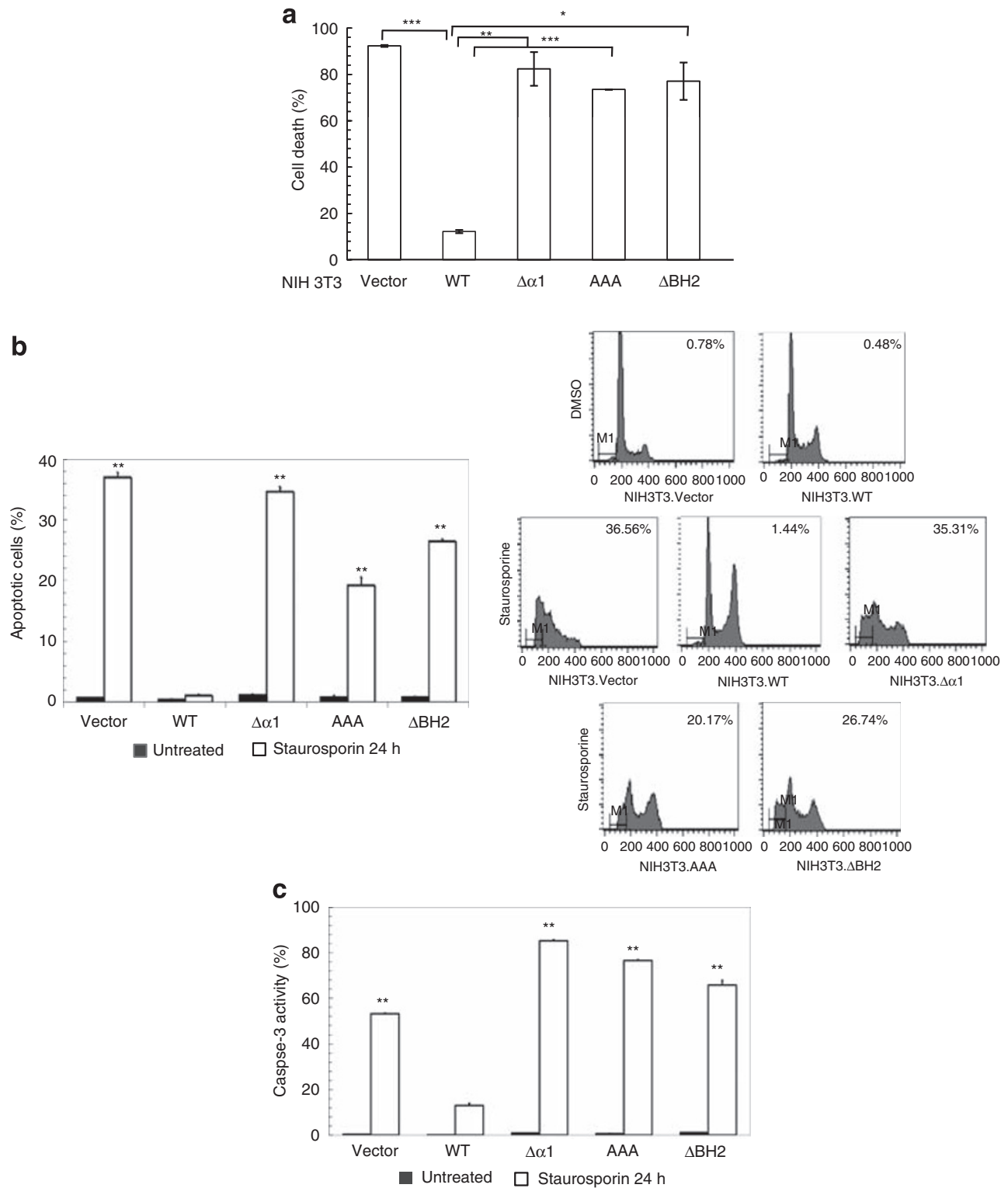

Figure 2 Anti-apoptotic activities of the Bcl-2 mutants. NIH3T3 cells stably expressing the WT or mutant forms of Bcl-2 were treated with DMSO (control) or staurosporine for $24 \mathrm{~h}$, then assayed for cell viability by trypan blue exclusion assay (a), for apoptosis (sub-G1 staining) by PI staining (b), or for caspase-3 activation using flow cytometry (c). Data represents mean \pm S.D. of combined results from three independent experiments. The M1 gate in (b) marks the population of cells with a sub-G1 DNA content. ${ }^{\star} P<0.01 ;{ }^{* \star} P<0.001 ;{ }^{* \star *} P<0.0001$. PI, propidium iodide

represents decreased autophagy, rather than an accelerated degradation of $\mathrm{LC}^{-} \mathrm{II}^{+}$autophagosomes, we assayed LC3-II in the presence of bafilomycin $A_{1}$, under rapamycin conditions (Figure $3 b$ ). Bafilomycin $A_{1}$ is a specific inhibitor of vacuolar $\mathrm{H}^{+}$-ATPase, which has been shown to cause accumulation of autophagosomes by blocking autophagosome-lysosome fusion. ${ }^{21}$ As expected, bafilomycin $\mathrm{A}_{1}$ elevated LC3-II levels in WT- and $\Delta \alpha 1$-expressing cells, demonstrating that blockage of autophagy by Bcl-2WT and $\mathrm{Bcl}-2 \Delta \alpha 1$ occurs at a stage of upstream autophagosome formation but not downstream autophagosome-lysosome fusion (Figure 3b). The p62 degradation is also used to evaluate autophagic flux, ${ }^{22}$ as p62 is a selective substrate of autophagy whose protein level is inversely related to the overall autophagic activity. ${ }^{22,23}$ Again, we found that the steady level of p62 was markedly upregulated in WT- and $\Delta \alpha 1$-expressing cells compared with cells expressing vector, $\triangle \mathrm{BH} 2$ or AAA (Figure 3b). Rapamycin treatment led to an evident increase of p62 degradation, which was subsequently reversed by bafilomycin $A_{1}$ (Figure $3 b$ ). The inverse relationship between the reduced LC3-II levels and the increased p62 levels in the $\mathrm{Bcl}-2 \Delta \alpha 1$ cells suggests that autophagic flux is attenuated by Bcl- $2 \Delta \alpha 1$, similarly to what is seen with WT. Thus, the $\alpha 1$ helix of $\mathrm{Bcl}-2$, which is required for the anti-apoptotic function, is dispensable for Beclin1 binding and autophagy inhibition, suggesting that $\mathrm{Bcl}-2$ can 
a
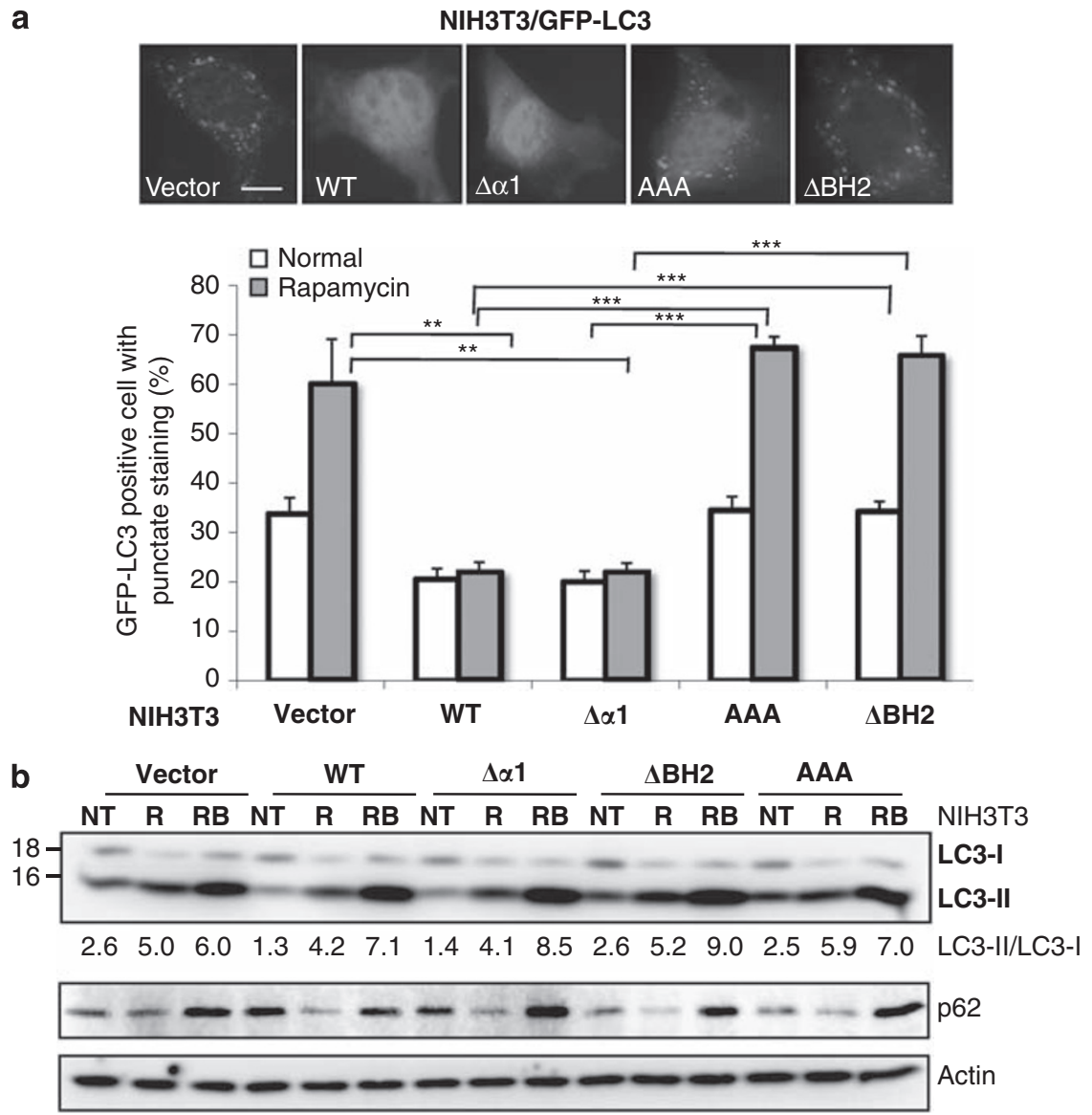

c

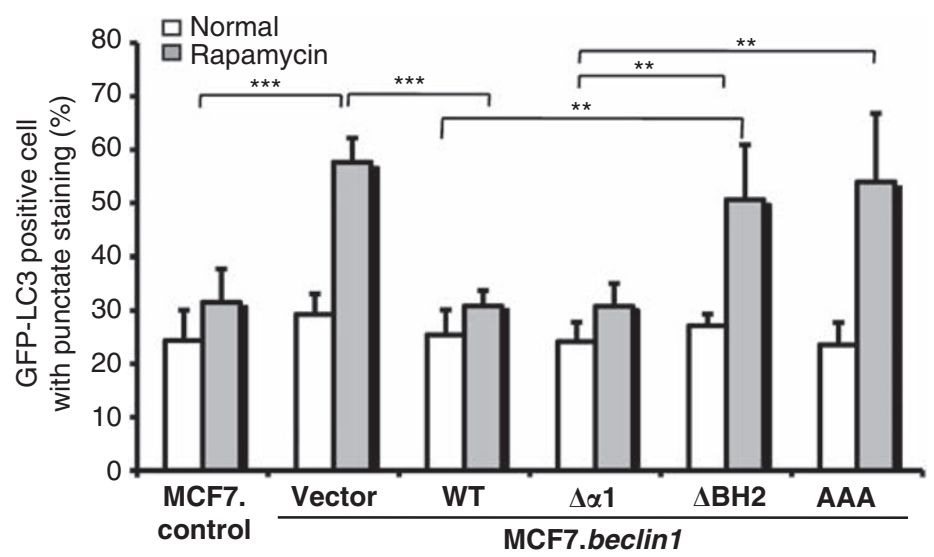

Figure 3 Anti-autophagic activity of the Bcl-2 mutants. (a) NIH3T3 cells expressing the WT or mutant Bcl-2 proteins as indicated were transfected with GFP-LC3 then treated with $2 \mu \mathrm{M}$ rapamycin for $6 \mathrm{~h}$. GFP-LC3 signal was detected using an inverted fluorescence microscope (top). Autophagy was quantified (percentage of cells with GFP-LC3 puncta) as mean \pm S.D. of the combined results from three independent experiments (bottom). Scale bar, $5 \mu$ m; ${ }^{* \star} P<0.01 ;{ }^{* \star *} P<0.001$. (b) NIH3T3 cells as described in (a) were cultured in a complete medium or treated with $2 \mu \mathrm{M}$ rapamycin with or without $100 \mathrm{nM}$ bafilomycin $\mathrm{A}_{1}$ for $2 \mathrm{~h}$. The cell lysates were subject to immunoblotting with a LC3 (top), a p62 (middle), and an actin (bottom) antibodies. Densitometric quantification of the LC3-II/LC3-I ratios under the indicated conditions is shown at the bottom of the LC3 blot. Similar results were obtained from three independent experiments. NT, no treatment; R, rapamycin treatment; RB, rapamycin and bafilomycin $\mathrm{A}_{1}$ treatment. (c) MCF7.control cells and MCF7.beclin 1 cells stably expressing the WT or mutant forms of Bcl-2 were transfected with GFP-LC3. At 18h posttransfection, cells were treated with $2 \mu \mathrm{M}$ rapamycin for $6 \mathrm{~h}$. Autophagy was quantified as mean \pm S.D. of the combined results from three independent experiments. ${ }^{\star \star} P<0.001 ;{ }^{* \star *} P<0.0001$

modulate autophagy through its interaction with Beclin1 in an anti-apoptosis-independent manner.

Previous studies showed that beclin1 deficiency increases one's propensity for breast cancer. ${ }^{11,24}$ We next assessed the effects of the $\Delta \alpha 1$ and other Bcl-2 mutants on Beclin1dependent autophagy in MCF7 cells. The MCF7 cells express undetectable levels of endogenous Beclin1 and are reluctant to induce autophagy upon starvation unless Beclin1 is 
overexpressed. ${ }^{11}$ Consistent with previous studies, the restoration of Beclin1 expression in MCF7 cells (MCF7. beclin1) led to a strong activation of autophagy upon rapamycin treatment (Figure $3 \mathrm{c}$ ). As seen in 3 T3 cells, the expression of $\mathrm{Bcl}-2 \Delta \alpha 1$ in MCF7.beclin1 cells inhibited Beclin1-mediated autophagy as effectively as WT with a significant reduction in the GFP-LC3 puncta and in the production of LC3-II (Figure $3 c$ and Supplementary Figure S2b). Similarly, treatment of the cells with bafilomycin $A_{1}$ causes a significant accumulation of LC3-II (Supplementary Figure S2b). A marked increase in the steady levels of p62 was also observed in cells expressing WT and Bcl-2 $2 \alpha 1$, but not in cells expressing AAA mutant (Supplementary Figure $\mathrm{S} 2 \mathrm{~b})$. Although the levels of p62 in vector and AAA-expressing MCF7.beclin1 cells are decreased by rapamycin treatment, the decrease was readily suppressed in the WT and Bcl-2 $2 \alpha 1$ cells (Supplementary Figure $\mathrm{S} 2 \mathrm{~b}$ ). In contrast, the $\triangle \mathrm{BH} 2$ and AAA mutants were defective in the inhibition of Beclin1mediated autophagy, despite having a similar level of expression as that of WT (Supplementary Figure S2a). Thus, the $\alpha 1$ helix is not essential for suppressing Beclin1-mediated autophagy nor for Beclin1 binding, reflecting a striking correlation between the ability of Bcl-2 to bind Beclin1 and its protection from Beclin1-mediated autophagy in both normal and cancerous cells.

To further confirm that the $\mathrm{Bcl}-2 \Delta \alpha 1$-mediated antagonism of autophagy is independent of its anti-apoptosis status, we subjected the MCF7.beclin1 cells expressing WT or the mutant $\mathrm{Bcl}-2$ to STS treatment. Apoptosis was measured by TUNEL staining. Unlike WT, the Bcl-2 $\Delta \alpha 1$ mutant failed to protect cells from STS-induced apoptosis, as was also seen with $\mathrm{Bcl}-2 \triangle \mathrm{BH} 2$ and $\mathrm{Bcl}-2 \mathrm{AAA}$ (Supplementary Figure S2c). Taken together, these results clearly demonstrate that the deletion of the $\alpha 1$ helix in Bcl-2 that abolished apoptotic inhibition generally has little or no effect on Bcl-2 antiautophagic activity. As summarized in Table 1, Bcl-2mediated antagonism of autophagy can be structurally and functionally separated from its previously defined antiapoptosis function, allowing us to evaluate its functional contributions to tumorigenesis in vivo.

The anti-apoptosis-defective but anti-autophagycompetent $\mathrm{Bcl}-2 \Delta \alpha 1$ enhances the tumorigenic properties of breast cancer cells. Identification of the $\mathrm{Bcl}-2 \Delta \alpha 1$ mutant allows us to examine the phenotypic consequence of the anti-autophagic aspect of $\mathrm{Bcl}-2$ in cancer without being affected by its inherent anti-apoptotic role. To test the hypothesis that the Beclin1-Bcl-2 interaction has an important role in Bcl-2 oncogenicity, we used the MCF7.beclin1 cells, which were previously used to demonstrate the tumor suppressor function of Beclin1, ${ }^{11}$

Table 1 Summary of the anti-autophagic and anti-apoptotic activities of the Bcl-2 mutants

\begin{tabular}{lcccc}
\hline Bcl-2 & Beclin1 & Bax/Bak & Anti-autophagy & Anti-apoptosis \\
\hline WT & + & + & + & + \\
$\Delta \alpha 1$ & + & - & + & - \\
AAA & - & - & - & - \\
$\Delta \mathrm{BH} 2$ & - & - & - & - \\
\hline
\end{tabular}

and assessed the effect of WT $\Delta \alpha 1$ and other mutant Bcl-2 proteins on the ability of Beclin1 to suppress cellular proliferation and transformation in MCF7 cells. As previously shown, ${ }^{11}$ the restoration of Beclin1 effectively blocked the hyperproliferation of MCF7 cells (Figure 4a). Surprisingly, the expression of Bcl-2 $2 \alpha 1$, which antagonizes Beclin1 function, stimulated cell proliferation of MCF7. beclin1 cells to a similar degree to that of WT Bcl-2. In contrast, $\mathrm{Bcl}-2 \Delta \mathrm{BH} 2$, which fails to bind Beclin1, did not enhance the growth rate of MCF7.beclin1 cells (Figure 4a). In-line with this, Bcl-2 $\Delta \alpha 1$-expressing cells formed more colonies then the control when plated at low density, and improved anchorage-independent growth in soft agar as efficiently as Bcl-2WT-expressing cells (Figure $4 \mathrm{~b}$ and $\mathrm{c}$ ). In contrast, $\triangle \mathrm{BH} 2$ - and $\mathrm{AAA}$-expressing cells were impaired in both regards (Figure $4 \mathrm{~b}$ and $\mathrm{c}$, and data not shown).

To further determine the effect of $\mathrm{Bcl}-2 \Delta \alpha 1$ on tumor growth in vivo, we orthotopically injected the Bcl-2-expressing MCF7.beclin1 cells into the mammary fat pad of nude mice and monitored the mice for tumorigenesis. Consistent with previous findings, ${ }^{11}$ restoring Beclin1 in MCF7 cells substantially inhibited tumor growth with tumor sizes eightfold smaller than the mean size of MCF7 control-derived tumors (Figure $4 \mathrm{~d}$ ). Bcl-2 $\Delta \alpha 1$ accelerated tumor growth to a level similar to that observed in Bcl-2WT-expressing cells-inoculated mice, whereas Bcl-2AAA defective in anti-autophagy failed to do so (Figure 4d). The tumor-promoting activity of $\mathrm{Bcl}-2 \Delta \alpha 1$ is further illustrated by tumor incidence: such as WT, more than $80 \%$ of the mice injected with the $\mathrm{Bcl}-2 \Delta \alpha 1$ cells developed tumors, whereas only $30 \%$ of the mice injected with the vector- or AAA-expressing cells developed tumors (Figure 4e). These data indicate that the expression of $\mathrm{Bcl}-2 \Delta \alpha 1$ renders breast cancer cells growth advantage in a manner that is independent of apoptosis inactivation.

To confirm that the observed activity of $\mathrm{Bcl}-2 \Delta \alpha 1$ is not because of ectopic expression of Beclin1 in MCF7 cells, we assayed MDA-MB-231 cells with normal Beclin1 levels and high basal levels of autophagy, ${ }^{25}$ stably transfected with WT or mutant Bcl-2 at equivalent levels (Supplementary Figure S3). Expression of Bcl-2 $2 \alpha 1$ considerably enhanced both lowdensity colony formation and growth of MDA-MB-231 cells as effectively as WT, whereas $\mathrm{Bcl}-2 \triangle \mathrm{BH} 2$ and $\mathrm{Bcl}-2 \mathrm{AAA}$ had minimal effects (Figure $5 a$ and $b$ ). Accordingly, the tumorigenic properties of MDA-MB-231 cells were also enhanced by WT and $\mathrm{Bcl}-2 \Delta \alpha 1$ in vivo (Figure $5 \mathrm{c}$ ). The mean tumor volume increased about sixfold in mice injected with MDA-MB231. Bcl-2 and MDA-MB-231.Bcl-2 $2 \alpha 1$ cells compared with mice injected with MDA-MB-231 cells-expressing vector, $\triangle \mathrm{BH} 2$, or AAA mutant (Figure $5 \mathrm{c}$ ). These data indicate that the anti-apoptosis defective $\mathrm{Bcl}-2 \Delta \alpha 1$ mutant, such as $\mathrm{Bcl}-2 \mathrm{WT}$, improves the transformed growth of MDA-MB-231 breast cancer cells.

Effect of Bcl-2 $2 \Delta \alpha 1$ on the transformation properties of breast cancer cells with low Beclin1 expression. To further address whether blockage of Beclin1 function is truly required for the oncogenic effects elicited by $\mathrm{Bcl}-2 \Delta \alpha 1$ and WT in MCF7 cells, we examined MCF7 cells with a monoallelic deletion of beclin1 and thus express low or undetectable levels of endogenous Beclin1. ${ }^{11}$ In particular, 


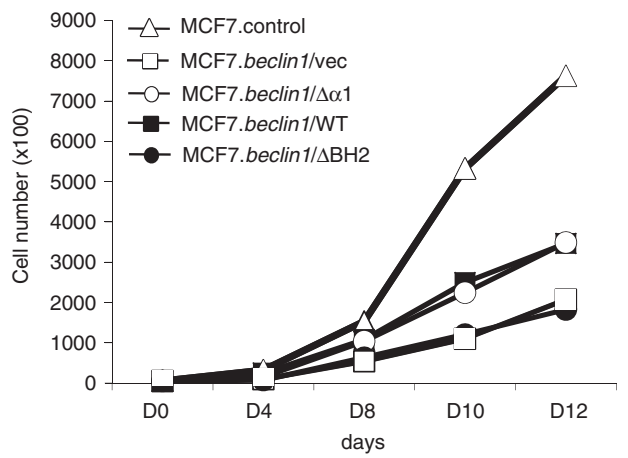

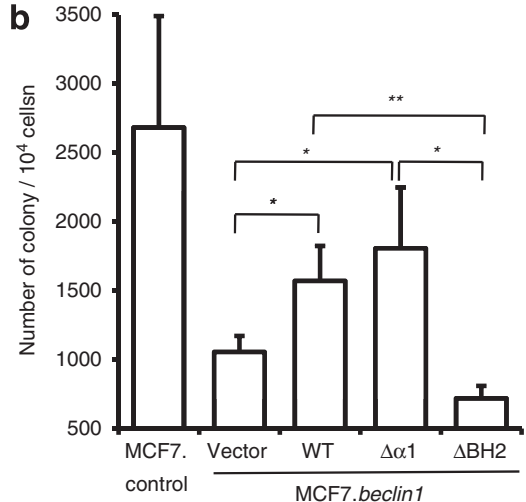

C
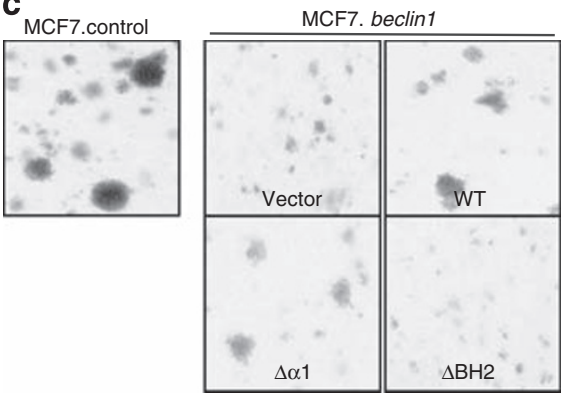

d

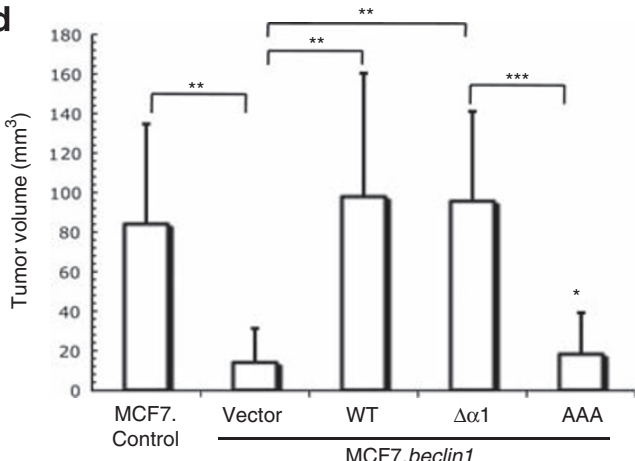

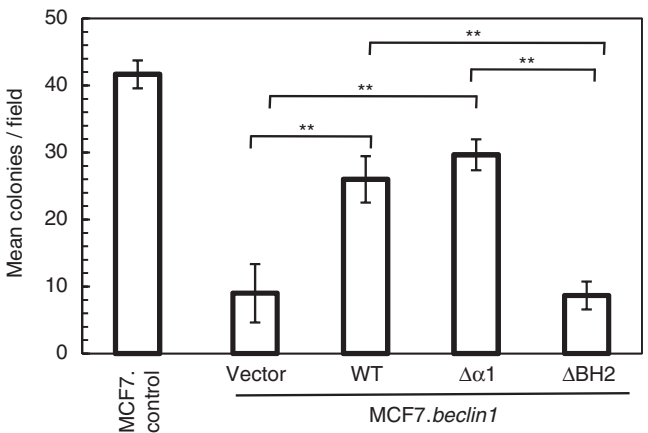

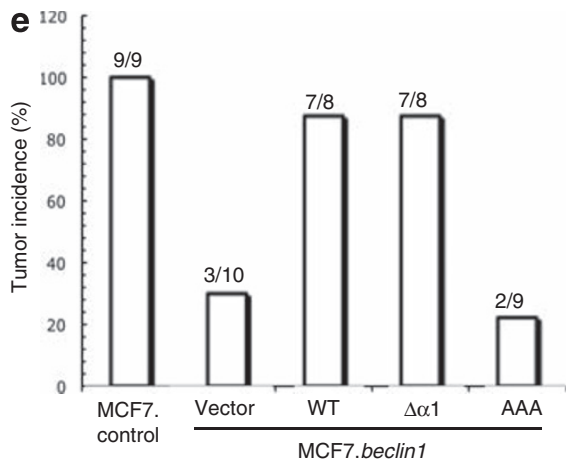

Figure 4 Effects of expression of the Bcl-2 mutants on the transformation property and tumorigenicity of MCF7.beclin 1 cells. (a) Cellular proliferation of MCF7.control cells (open triangles), MCF7.beclin1 cells stably expressing empty vector (open squares), WT Bcl-2 (closed squares), Bcl-2 $\Delta \alpha 1$ (open circles), and Bcl-2 $\Delta \mathrm{BH} 2$ (closed circles). Representative result from three independent experiments is shown. (b) Clonogenic assay in vitro. MCF7 cells in (a) were plated at low density ( $2500 \mathrm{cells}$ per $10 \mathrm{~cm}$ plate), grown for 7 days and fixed and stained with crystal violet. Data represent mean \pm S.D. of duplicate plates from three independent experiments. (c) Anchorage-independence. MCF7 cells $\left(1 \times 10^{4}\right)$ in (a) were incubated in soft-agar as described in Materials and Methods. After 3 weeks, colonies were photographed and counted. Colony numbers were quantified as means \pm S.D. of pooled results from three independent experiments. (d and e) Tumorigenicity. MCF7 cells as indicated were injected into the breast pad of nude mice $\left(5 \times 10^{6}\right.$ cells/injection), and tumor size and tumor incidence were measured at 21 days postinoculation. Numbers on top of bar (e) denote the number of autopsy-confirmed tumors at 21 days per number of mice injected with each cell line. ${ }^{*} P<0.05 ;{ }^{*} P<0.01 ;{ }^{* \star *} P<0.001$

we constructed MCF7 cell lines stably expressing WT or mutant $\mathrm{Bcl}-2$ variants at similar levels (Supplementary Figure S4c). Upon injection into mice, MCF7 cells expressing Bcl-2 gave rise to tumors that were three times larger than in controls, which is less significant compared with the 10-fold increase seen in their MCF7.beclin1 counterparts (Supplementary Figure S4a and S4b). In-line with this, Bcl$2 \Delta \alpha 1$ expression only modestly increased xenotransplanted tumor growth of MCF7 cells compared with the vector control, $\mathrm{Bcl}-2 \Delta \mathrm{BH} 2$, and $\mathrm{Bcl}-2 \mathrm{AAA}$ with no statistically significant difference observed in repeated experiments
(Supplementary Figure S4a). Hence, the enhanced transformation property derived from WT, and particularly the $\Delta \alpha 1$ mutant, is severely blunted if Beclin 1 expression is low. Of note, we also found that WT Bcl-2-mediated transformation was only partially abrogated by low Beclin1 levels when compared with Bcl-2 $2 \alpha 1$ (Supplementary Figure S4). This observation suggests that although Bcl-2 confers oncogenicity by targeting the Beclin1-autophagy protein, the full capacity of $\mathrm{Bcl}-2$ to foster breast tumor growth may extend beyond its ability to inhibit Beclin1; other mechanisms may also be involved including apoptosis inhibition. 

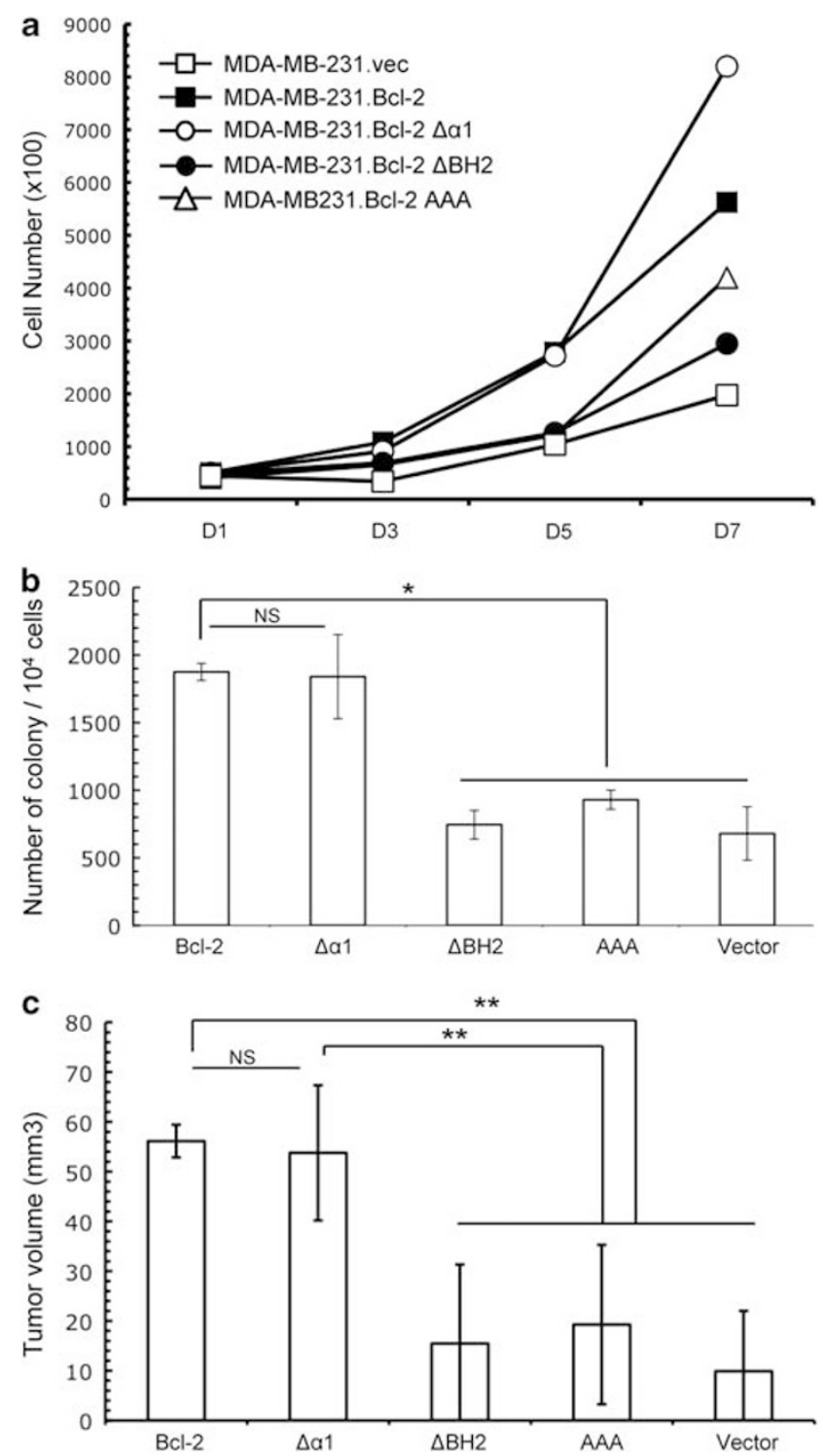

Figure 5 Effects of expression of the Bcl-2 mutants on the transformation property and tumorigenicity of MDA-MB-231 cells. (a) Cellular proliferation (left panel) of MDA-MB-231 cells stably expressing empty vector (open squares), WT Bcl-2 (closed squares), Bcl-2 $\Delta \alpha 1$ (open circles), Bcl-2 $\Delta \mathrm{BH} 2$ (closed circles), and $\mathrm{Bcl}-2 \mathrm{AAA}$ (open triangles). Representative result from three independent experiments is shown. (b) Clonogenic assay in vitro. MDA-MB-231 cells in (a) were plated at low density ( 2500 cells per $10 \mathrm{~cm}$ plate), grown for 7 days and fixed and stained with crystal violet. Data represent mean \pm S.D. of duplicate plates from three independent experiments. ${ }^{*} P<0.05$; NS, not significant. (c) Tumorigenicity. MDA-MB-231 cells as indicated were injected into the breast pad of nude mice $\left(10^{7}\right.$ cells/injection), and tumor size were measured at 30 days post-inoculation. Data represent mean \pm S.D. from three independent experiments. ${ }^{* \star} P<0.01$; NS, not significant

These results reinforced observations made from an in vitro soft agar colony-forming assay. Unlike MCF7.BCl-2WT cells, which formed colonies in soft agar with higher efficiency and of larger sizes, MCF7.Bcl-2 $2 \alpha 1$ cells were impaired in their clonigenicity in vitro, to a degree close to those observed in the AAA- and $\triangle \mathrm{BH} 2$-expressing MCF7 cells (Supplementary Figure S4b). Taken together, these results indicate that the inhibition of Beclin1 is necessary for $\mathrm{Bcl}-2 \Delta \alpha 1$-facilitated transformation of breast cancer cells and that Beclin1mediated autophagy serves as an important mechanism for preventing the deregulated growth of breast tumor cells, at least in certain contexts.

Bcl-2 $\alpha \alpha 1$ suppresses autophagy in breast tumor xenografts in vivo. We next tested whether the enhanced tumor growth induced by Bcl-2 $2 \alpha \alpha$, as shown in Figure $4 d$, is virtually associated with altered differentiation and autophagy in vivo. To address this question, WT- and mutant $\mathrm{Bcl}-2$ expressing MCF7.beclin1 xenograft tumors were examined by histology and electron microscopy. The general morphology of the xenografts was similar, being primarily composed of poorly differentiated adenocarcinoma with irregular nuclei, periphery lymphoid infiltration, and frequent blood vessels (Figure 6a). Notably, large areas of necrosis and multinucleated cells were evident in most of the WT and the $\Delta \alpha 1$ tumor sections, but not in the vector control and AAA tumor sections (Figure $6 \mathrm{a}$ ). Consistent with their increased proliferation in vitro, the WT- and the $\Delta \alpha 1$-expressing tumors had an approximately two-fold increase $($ mean $=60)$ in the number of mitotic figures per 10 high-power fields compared with the vector and the AAA tumors (mean $=24$ ) (Figure $5 b$ ). All Bcl-2-expressing tumors maintained a high level of $\mathrm{Bcl}-2$ as detected by the anti-HA IHC staining (Figure 6c), confirming the stability of ectopically expressed $\mathrm{Bcl}-2$ in vivo. This data suggests that the impairment of autophagy by $\mathrm{Bcl}-2 \Delta \alpha 1$ increases mitotic rate and proliferation in vivo and, thus, is preserved from Bcl-2WT in an antiapoptosisdefective background.

To further evaluate whether $\mathrm{Bcl}-2 \Delta \alpha 1$ reduces autophagy in breast cancer xenografts in vivo, the tumor tissue sections were subject to electron microscopy analysis. Our results show that the expression of Bcl-2 $2 \Delta \alpha 1$ and WT led to a marked reduction of the number of autophagic vacuoles in tumors (Figure 6d). However, tumors expressing the anti-autophagy defective AAA mutant exhibited high levels of autophagy similar to the vector control. These data indicate that $\mathrm{Bcl}-2 \Delta \alpha 1$ expression reduces autophagic activity that is associated with increased tumorigenesis in vivo.

Accumulation of p62 and DNA damage in $\mathrm{Bcl}-2 \Delta \alpha 1$ breast tumor xenografts. To further investigate how Bcl-2's inhibition of autophagy affects tumor growth in vivo, tumors generated by $\mathrm{Bcl}$-2-expressing breast cancer cells were examined for p62 accumulation, unfolded protein response (UPR), and DNA damage activation, all of which are strongly associated with impaired autophagy in previous studies. ${ }^{14}$ As shown in Figure 7a, a marked increase of p62 was detected in $\mathrm{Bcl}-2 \Delta \alpha 1$-expressing tumors as well as in that of Bcl-2WT, but not in tumors derived from Bcl-2AAAexpressing cells (Figure $7 \mathrm{a}$ ). Unlike previous studies that showed a concurrent increase of p62 and ER chaperons in autophagy-deficient tumors, ${ }^{14}$ no evident difference in the levels of GRP78, an ER chaperon and an early marker for UPR, was detected between Bcl-2 tumor xenografts (Figure $7 \mathrm{~b}$ ). To investigate if $\mathrm{Bcl}-2 \Delta \alpha 1$ inhibition of autophagy also results in enhanced DNA damage as observed with beclin1-deficient breast tumors, ${ }^{14}$ the appearance of $\gamma$ - $\mathrm{H} 2 \mathrm{AX}$ 
a
MCF7. beclin1

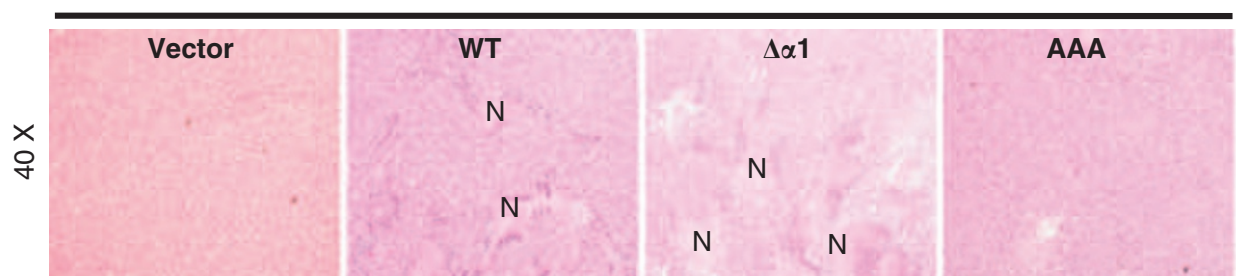

b

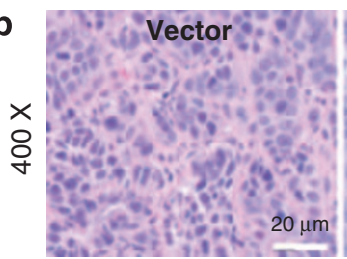

C

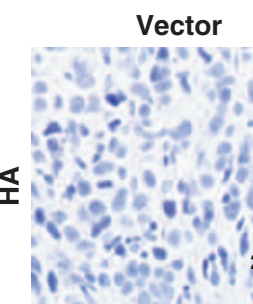

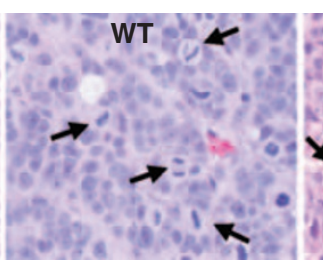

WT

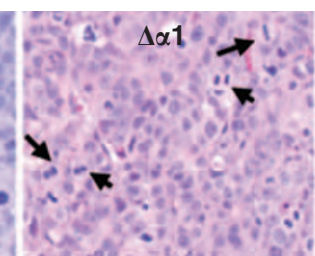

$\Delta \alpha 1$

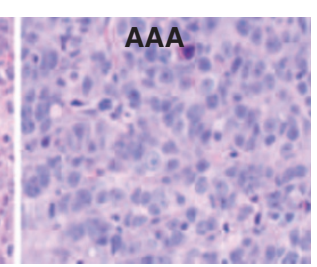

AAA
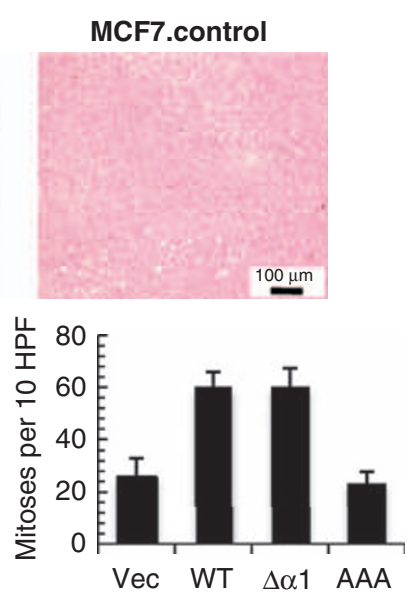
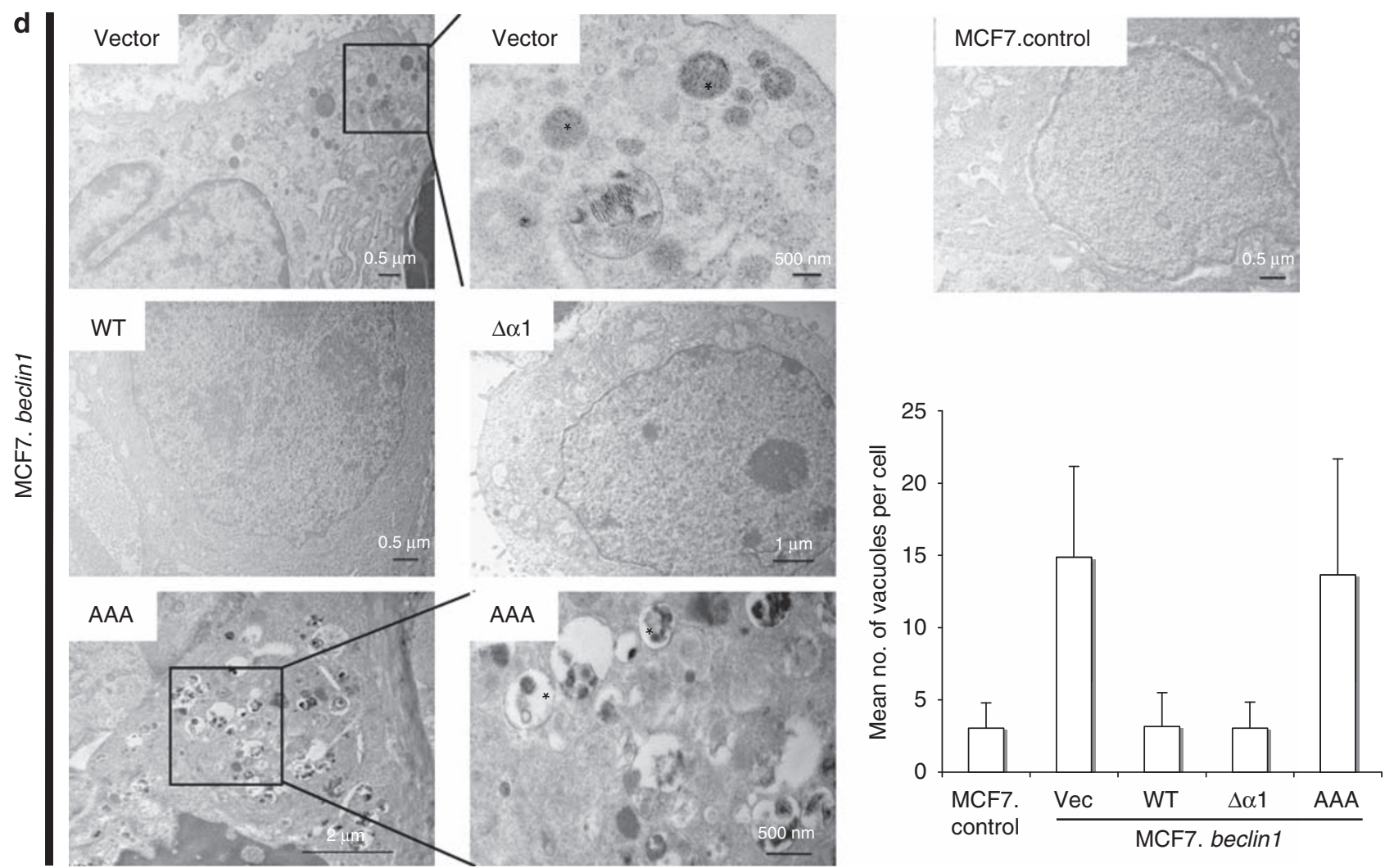

Figure $6 \mathrm{Bcl}-2 \Delta \alpha 1$ promotes tumor growth and suppresses autophagy in xenograft tumors in vivo. (a) Hematoxylin/eosin (H\&E) staining of xenograft tumors generated by the indicated MCF7 cell lines. Pronounced necrosis $(\mathrm{N})$ was detected in the Bcl-2 $\Delta \alpha 1$ tumor independent of the means of Bcl-2-mediated apoptosis inhibition. Original magnification, $\times 40$. (b) Mitotic activity was brisk in xenografts with WT and Bcl-2 $\Delta \alpha 1$ expression. Original magnification, $\times 400$. The number of mitotic figures per 10 highpower fields (HPF) in WT and Bcl-2 $\Delta \alpha 1$ xenografts were significantly higher than in vector and AAA xenografts. Arrows indicate mitotic cells. (c) Immunohistochemical analysis of HA-Bcl-2 (WT and mutants) expression in breast tumor sections developed from implanted MCF7.beclin 1 cells as indicated. (d) Electron microscopic analyses of xenograft tumors. Magnified images of the insets highlighted abundant autophagic vacuoles in tumors derived from the empty vector- and the AAA-expressing MCF7.beclin1 cells. The mean number of autophagic vacuoles per cell in tumors was determined (data are mean \pm S.D., $n=25$ ). Asterisks denote autophagic vacuoles (double- or single-membrane vacuolar structures containing recognizable cytoplasmic materials) counted in each sample 
a

Vector

WT

$\Delta \alpha 1$

AAA

$400 x$
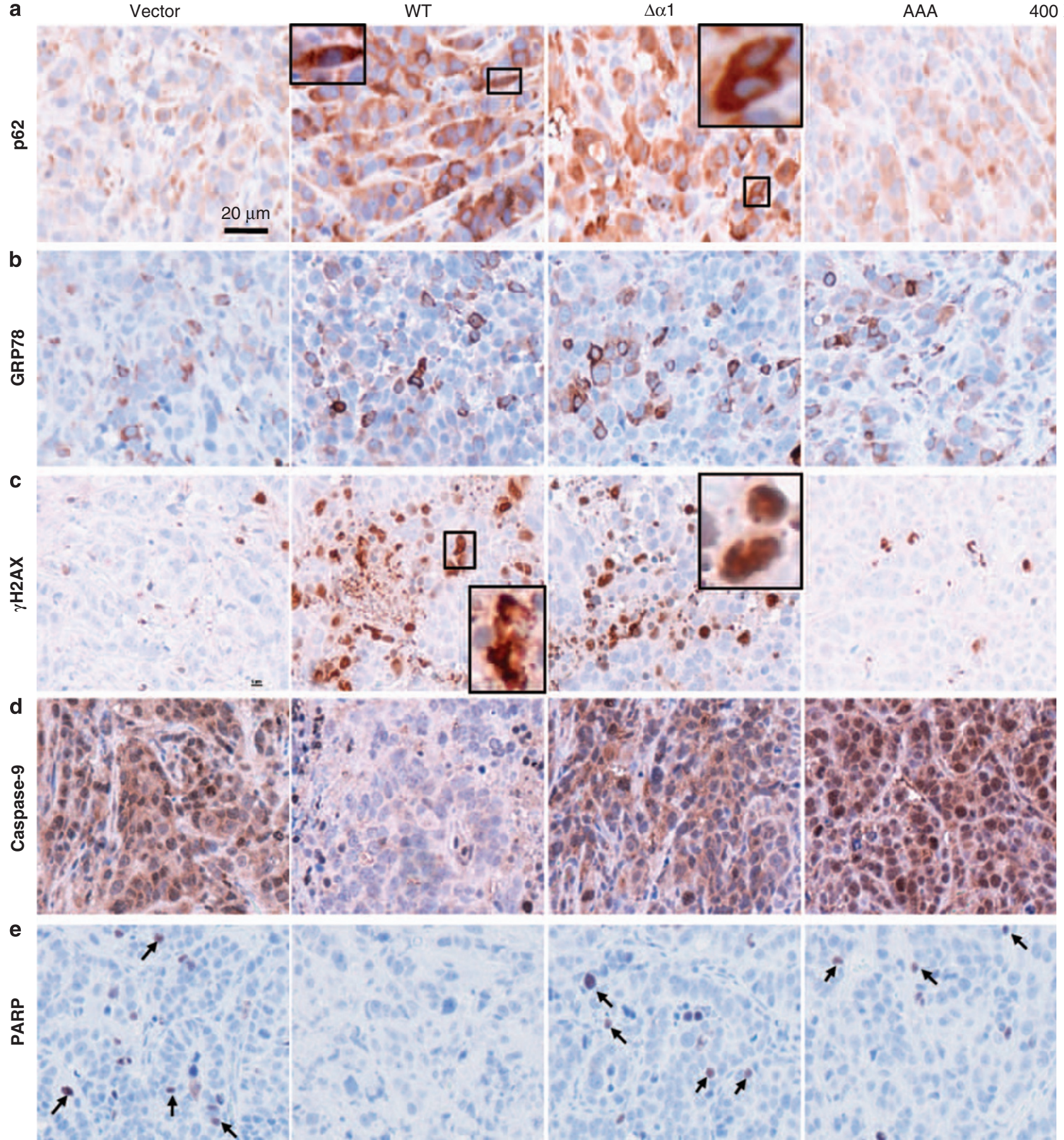

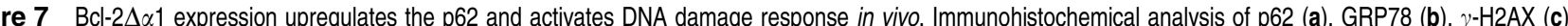
cleaved caspase-9 (d), and PARP (e) in breast tumor sections developed from implanted MCF7.beclin1 cells as indicated. The insets highlighted the staining of the indicated proteins. Original magnification, $\times 400$. Arrows indicate apoptotic cells with a positive PARP cleavage

nuclear foci staining, an important marker for DNA damage, was monitored in xenograft samples. Our results indicate that the $\mathrm{Bcl}-2 \Delta \alpha 1$ tumors exhibited increased $\gamma-\mathrm{H} 2 \mathrm{AX}$ foci staining reminiscent of that of WT, whereas only scattered $\gamma-\mathrm{H} 2 \mathrm{AX}$ staining was detected in the AAA mutant and vector tumors (Figure $7 \mathrm{c}$ ). Thus, these data imply that the autophagy attenuation and p62 accumulation due to $\mathrm{Bcl}-2 \Delta \alpha 1$ overexpression renders breast cancer cells prone to increased DNA damage, which may contribute to their pro-proliferative state in breast cancer.

To confirm that the increased p62 and $\gamma-\mathrm{H} 2 \mathrm{AX}$ staining associated with $\mathrm{Bcl}-2 \Delta \alpha 1$ is due to the suppression of 
autophagy without preventing apoptosis, we evaluated the apoptosis status of xenograft tumors. Large regions of the cleaved caspase- 9 immunoreactivity were detected in tumors expressing Bcl-2 $\Delta \alpha 1$ and Bcl-2AAA, whereas scant caspase9 activation was found in WT Bcl-2 tumors (Figure 7d). Furthermore, the cleavage of PARP, an event downstream of caspase activation and a biomarker of the end-stage of apoptosis, was frequent in the $\Delta \alpha 1, \mathrm{AAA}$, and vector tumors by IHC staining, but was rarely encountered in the Bcl-2WT tumors (Figure 7e). Taken together, our $\mathrm{Bcl}-2 \Delta \alpha 1$ data indicate that the inhibition of Beclin1 and hence autophagy by $\mathrm{Bcl}-2$ leads to the $\mathrm{p} 62$ accumulation and the activation of DNA damage responses, ultimately facilitating tumor growth in vivo independent from its role in antagonizing cellular apoptosis.

\section{Discussion}

Both apoptosis and autophagy are important tumor suppressor mechanisms. ${ }^{8}$ Although it has been well documented that apoptosis inhibition has an integral role in Bcl-2-mediated oncogenesis, the role of autophagy inhibition by $\mathrm{Bcl}-2$ in cancer remains largely unknown. In this study, we demonstrated for the first time that Bcl-2 promotes tumor growth by an anti-apoptosis-independent mechanism that jeopardizes the autophagy pathway in MCF7 and MDA-MB-231 breast cancer cells. Our finding thus indicates that Bcl-2-mediated inhibition of autophagy represents an important mechanism of oncogenesis. Previous studies have shown that mutations of Beclin1 that abolish its interaction with $\mathrm{Bcl}-2$ confer a gain-offunction phenotype in autophagy induction, as was also seen with silencing $b c l-2$ in breast cancer cells. ${ }^{6,15}$ However, in these studies, the significance of autophagy inhibition by $\mathrm{Bcl}-2$ in tumorigenesis is not yet known and delineating this is complicated by the fact that the two activities, anti-apoptosis and anti-autophagy, are intertwined and concomitantly propagated by Bcl-2. Our discovery that an antiautophagycompetent but antiapoptosis-disabled $\mathrm{Bcl}-2$ mutant promotes the tumorigenic properties of MCF7 breast cancer cells explicitly demonstrates that autophagy inhibition may have an equal, if not greater, impact on the ability of Bcl-2 to promote tumorigenesis and hence, should be merited as a novel mechanism by which Bcl-2 drives breast tumor growth.

$\mathrm{Bcl}-2$ is frequently implicated in cancers and is associated with the aggressive tumor phenotype. ${ }^{26}$ Our studies have allowed us to genetically dissect the specific contribution of autophagy regulation to the overall function of $\mathrm{Bcl}-2$ by constructing a Bcl-2 mutant that is able to block autophagy but is unable to inhibit apoptosis in breast cancer cells. This mutant is highly competent in promoting tumor growth in Beclin1-expressing breast cancer cells, suggesting that the Bcl-2-mediated inhibition of apoptosis is not the sole mechanism that confers tumor development, but rather that the Bcl-2-mediated blockage of Beclin1-dependent autophagy also participates in oncogenesis at significant level. Moreover, when the level of Beclin1 is low or deficient in MCF7 cells, the ability of this mutant to improve the transformation properties of breast cancer cells is also significantly reduced, further suggesting the importance of Beclin1 antagonism in Bcl-2-associated oncogenesis. This finding seems to be without precedent as no clear evidence has ever been provided on the oncogenic importance of autophagy dysregulation by $\mathrm{Bcl}-2$. Our data, however, do not rule out an important role for apoptosis inhibition in tumor growth but, instead, it identifies a role of Bcl-2-associated autophagy defect in oncogenesis. It is also worth noting that most tumor cells including MCF7 breast cancer cells are refractory to apoptosis because of the inactivation of crucial apoptotic effectors such as caspase- 3 in the case of MCF7 cells. Such an increased apoptotic threshold in tumor cells may benefit oncogenic transformation mediated by disabling autophagy. Analogous to Bcl-2, other predominant oncogenes, such as mTOR, AKT, and FLIP, provide constitutive signals to suppress autophagy, whereas many known tumor suppressors, such as PTEN, TSC1/TSC2, and p53, have been found to activate it, strongly suggesting that interfering with autophagic signaling and machinery represents a common oncogenic transformation strategy in cancer. ${ }^{8,9,27}$

The data presented here indicates that autophagy inhibition is not a 'passenger' function of $\mathrm{Bcl}-2$ but rather predominant in $\mathrm{Bcl}-2$-mediated oncogenesis. An important question that arises is why $\mathrm{Bcl}-2$ possesses separate mechanisms for oncogenesis and how autophagy deregulation contributes to tumor development. Although impaired apoptosis is recognized as a key step in tumorigenesis, it has been shown that anti-apoptosis is insufficient on its own to fully transform cells and that full-fledged malignancy often requires the synergism of multiple oncogenic events such as enhanced proliferation, genome instability, deregulation of cell cycle control, and senescence. ${ }^{28}$ Given the substantial contributions of autophagy to the quality control of cells by the removal of damaged proteins and organelles, it is probably not surprising that a defect in autophagy can lead to the accumulation of undesirable components, which can fuel inflammation and induce chromosomal instability, as has been recently demonstrated in the autophagy-deficient tumors and immortalized cells. $^{12-14}$ Consistent with this, we found that the Bcl-2 $2 \Delta \alpha 1$ mutant, which is sufficient to antagonize Beclin1-dependent autophagy, induces the p62 accumulation and increased DNA damage in breast cancer cell xenografts, whereas the Beclin1-binding deficient Bcl-2 mutant fails to do so. Thus, the $\mathrm{Bcl}-2$ antagonism of autophagy can function independently to contribute to tumor growth, at least under certain conditions. In this scenario, the increase of $\mathrm{Bcl}-2$ seen in most cancers may not only impart a survival advantage to these cells, but can also trigger oncogenic stress by compromising the quality control and metabolic equilibrium driven by autophagy.

In summary, we have demonstrated an important role for $\mathrm{Bcl}-2$ inhibition of autophagy in the transformation properties of MCF7 breast cancer cells. Beyond its established antiapoptotic functions, Bcl-2 targets the autophagy effector, Beclin1, and this activity subverts internal protein quality control and genome stability, promoting tumor cells growth. Our findings thus indicate that two tumor-suppressive pathways, autophagy and apoptosis, both targeted by $\mathrm{Bcl}-2$, actually have distinctive roles in protecting against cancer. Future studies will aim to understand how these two functions are regulated in different cancer conditions, which may lead to new strategies for cancer therapy. 


\section{Materials and Methods}

Cell culture. NIH3T3, 293T, and MDA-MB231 cells were cultured in Dulbecco's modified Eagle's medium (DMEM) supplemented with 10\% fetal bovine serum, $2 \mathrm{mM}$ L-glutamine, and $1 \%$ penicillin-streptomycin (Gibco-BRL, Carlsbad, CA, USA). The autophagy-deficient low-Beclin1-expressing MCF7 control, and MCF7.beclin1 cells were generously provided by Dr. B Levine and maintained in DMEM supplemented with 10\% Tet-system-approved FBS (Clontech, Mountain View, CA, USA), $300 \mu \mathrm{g} / \mathrm{ml}$ hygromycin and $1 \mu \mathrm{g} / \mathrm{ml}$ doxycycline, as described previously. ${ }^{11}$ Transient transfection was performed with Fugene 6 (Roche, Indianapolis, IN, USA), Lipofectanine 2000 (Invitrogen, Carlsbad, CA, USA), or Calcium phosphate (Clontech). NIH3T3, MCF7, and MDA-MB231 stable cell lines were established using a standard protocol of selection with $2 \mu \mathrm{g} / \mathrm{ml}$ of puromycin (Sigma-Aldrich, St. Louis, MO, USA).

Plasmid construction. The PCR-amplified Bcl-2 DNA was cloned into a modified pEF-IRES-puro vector (Invitrogen) encoding an N-terminal HA tag (pEF-HA-Bcl-2). Mutations in bcl-2 were generated by PCR (Hi-Fidelity PCR kit, Roche) with oligonucleotide-directed mutagenesis. Specifically, Bcl-2 $\Delta \alpha 1$ (lacking the $\mathrm{N}$-terminal 22 residues) was amplified from the $\mathrm{pEF}-\mathrm{HA}-\mathrm{Bcl}-2$ vector using specific primers; the $\mathrm{Bcl}-2 \Delta \mathrm{BH} 2$ (lacking residues $187-203$ of $\mathrm{BH} 2$ domain) mutant was created through two-step PCR mutagenesis. The Bcl-2 AAA mutant with alanine substitutions at the Trp144-Gly145-Arg146 residues was created using a Quickchange site-directed mutagenesis kit (Stratagene, Wilmington, DE, USA). All of the PCR products with the indicated Bcl-2 mutations were then cloned in frame into the Xhol/Mlul sites of the pEF-IRES-puro vector, for both transient and stable expression. All mutant constructs were completely sequenced to ensure the presence of the desired mutation and the absence of secondary mutations. Constructs expressing the epitope-tagged Bax and Bak were kindly provided by J. Marie Hardwick (John Hopkins University). The GST fusion protein of Bak (GST-Bak $\Delta$ TM) from $E$. coli and the Beclin1-V5 plasmid have been described previously. ${ }^{16}$ All constructs were sequenced using an ABI PRISM 377 automatic DNA sequencer.

Autophagy analyses. Quantitative GFP-LC3 light microscopy assay was performed in NIH3T3 and MCF7 stable cells expressing the WT or mutant forms of $\mathrm{BCl}-2$, then transfected with a GFP-LC3-expressing plasmid. ${ }^{19}$ Autophagy was then induced by starvation or rapamycin treatment. For starvation, the cells were washed three times with PBS and incubated in Hank's solution (Invitrogen) for $4 \mathrm{~h}$ at $37^{\circ} \mathrm{C}$. Alternatively, the cells were cultured in DMEM containing $1 \%$ FBS and $2 \mu \mathrm{M}$ rapamycin (Sigma-Aldrich) for 2-6h. For autophagic flux, the rapamycin-treated cells were cultured in DMEM containing $100 \mathrm{nM}$ Bafilomycin $\mathrm{A}_{1}$ for $2 \mathrm{~h}$. LC3 mobility shift and the p62 level were detected by immunoblotting as previously described. ${ }^{29}$

Apoptosis analyses. NIH3T3 and MCF7 cells stably expressing the WT or mutant forms of the Bcl-2 were seeded at $1 \times 10^{6}$ cells per well into 6 -well plates for $24 \mathrm{~h}$. The cells were then treated with fresh medium containing $1 \mu \mathrm{M}$ Staurosporine for up to $24 \mathrm{~h}$. Overall cell viability was measured by trypan blue exclusion assay. For the analysis of apoptotic cells, the samples were prepared using an DEADEND Fluorometric TUNEL system kit (Promega, Madison, WI, USA) according to the manufacturer's instructions. Nuclei were counterstained with 4, 6-diamidino-2 phenylindole (DAPI). Fluorescence microscopy analyses were performed with an Olympus IX-70 microscope. The percentage of TUNEL-positive cells was determined against the number of DAPI-stained nuclei. For the sub-G1 PI staining assay, the cells were collected with the cell dissociation buffer (SigmaAldrich) and then fixed with $70 \%$ of ethanol overnight at $-20^{\circ} \mathrm{C}$. Fixed cells were washed twice with PBS, and incubated in PBS containing propidium (PI; $5 \mu \mathrm{g} / \mathrm{ml}$ ), RNase A (1 mg/ml), and Triton X-100 $(0.5 \%)$ at room temperature for $30 \mathrm{~min}$. Fluorescence emitted from the propidium-DNA complex was measured using FACScan flow cytometry. Cells with a sub-G1 content were identified as apoptotic. The data was analyzed using Cell Quest (BD Bioscience, Franklin Lakes, NJ, USA). For caspase-3 activity assay, the cells were harvested after treatment, washed three times with PBS and fixed with fixation medium (Invitrogen, Catalog no. GAS001S) for $15 \mathrm{~min}$, permeabilized with permeabilization medium (Invitrogen, Catalog no. GAS002S) for another $15 \mathrm{~min}$, and then stained with PE-conjugated anti-Caspase-3 active form (BD biosciences, no. 550821) for flow cytometry analysis. Data were analyzed by FlowJo-6.4 (TreeStar, San Carlos, CA, USA).

Immunoblotting, immunoprecipitation and GST pulldown. For immunoblotting, the polypeptides were resolved by SDS-PAGE and transferred onto a PVDF membrane (Bio-Rad, Hercules, CA, USA). The membranes were blocked with $5 \%$ nonfat milk, and probed with the indicated antibodies. Goat antibodies coupled to horseradish peroxidase specific to mouse or rabbit immunoglobulins were used as secondary antibodies (diluted 1:10000, SigmaAldrich). Immunodetection was achieved with a chemiluminescence reagent (Pierce, Rockford, IL, USA) and detected by LAS 3000 (Fuji Film) (Fuji Film Co., Tokyo, Japan).

For immunoprecipitation, cells were harvested and then lysed in a 1\% NP40 lysis buffer supplemented with complete protease inhibitor cocktail (Roche). After preclearing with protein $A / G$ agarose beads for $2 \mathrm{~h}$ at $4^{\circ} \mathrm{C}$, whole-cell lysates were used for immunoprecipitation with the indicated antibodies. Generally, $2 \mu \mathrm{g}$ of the commercial antibodies was added to $1 \mathrm{ml}$ of the cell lysate, which was then incubated at $4^{\circ} \mathrm{C}$ for 8-12 h. After addition of protein $A / G$ agarose beads, incubation was continued for another $2 \mathrm{~h}$. Immunoprecipitates were extensively washed with an NP40 lysis buffer and eluted with an SDS-PAGE loading buffer by boiling for $5 \mathrm{~min}$.

For in vitro GST pulldown assay, GST by itself or a GST-Bak $\Delta$ TM fusion protein was purified from E.coli strain BL21 (DE3) (Promega). 293T cell lysates were incubated with glutathione beads containing the GST fusion protein in a binding buffer (20 mM HEPES (pH 7.4), $100 \mathrm{mM} \mathrm{NaCl}, 1 \% \mathrm{NP}-40$, and protease inhibitors) for $2 \mathrm{~h}$ at $4^{\circ} \mathrm{C}$. The glutathione beads were then washed four times with the binding buffer, and the proteins associated with the beads were analyzed by SDS-PAGE and subjected to immunoblot assay with the phosphorimager.

Immunofluorescence and confocal laser scanning microscopy. NIH3T3 stable cells grown on 8-well chamber slides were fixed with $2 \%(\mathrm{w} / \mathrm{v})$ paraformaldehyde in PBS for $20 \mathrm{~min}$, permeabilised with $0.2 \%(\mathrm{v} / \mathrm{v})$ Triton X-100 for $15 \mathrm{~min}$ and blocked with $10 \%$ goat serum (Gibco-BRL) for $1 \mathrm{~h}$. Primary antibody staining was performed using antiserum or purified antibodies in $1 \%$ goat serum for $1-2 \mathrm{~h}$ at room temperature. The cells were then extensively washed with PBS and incubated with diluted secondary antibodies in $1 \%$ goat serum for $1 \mathrm{~h}$. The cells were mounted using Vectashield (Vector Laboratories, Inc., Burlingame, CA, USA). The confocal images were acquired using a Nikon Eclipse $\mathrm{C} 1$ laser-scanning microscope (Nikon Instruments Inc., Melville, NY, USA) fitted with a $\times 60$ Nikon objective and Nikon Element image software (Nikon Instruments Inc.).

Clonogenic assay in vitro. Engineered MCF7 and MDA-MB-231 cells were plated at low density ( 2500 cells per $10 \mathrm{~cm}$ plate), grown for 10 days, and then fixed and stained with crystal violet with colony numbers counted.

Characterization of cell growth in vitro. Engineered MCF7 and MDA-MB231 cells $\left(1 \times 10^{5}\right)$ were plated into 6 -well microtiter plates. Cell numbers were counted with a hemocytometer (Beckman Coulter, Brea, CA, USA) at each time point for 12 days. Cell counts were performed in triplicate. One typical experiment was shown.

Soft agar anchorage-independent growth assay. To evaluate anchorage-independent colony formation, MCF7 cells as indicated $\left(1 \times 10^{4}\right)$ were suspended in complete medium containing $0.3 \%$ Nobel agar (Difco, Lawrence, $\mathrm{KS}$, USA) supplemented with $2 \mu \mathrm{g} / \mathrm{ml}$ puromycin and plated in 6-well plates over a basal layer of complete medium containing $0.5 \%$ agar. Colonies were scored 21 days after plating and were photographed by phase-contrast microscopy. Images were captured with the QCapture software program (Olympus, Center Valley, PA, USA). Clonogenicity was determined in triplicate experiments.

In vivo tumorigenicity assay. To measure in vivo tumorigenicity, MCF7, MCF7.beclin1, and MDA-MB-231 breast tumor cells expressing WT or mutant forms of Bcl-2 $\left(5 \times 10^{6}\right)$ were transplanted into the mammary fat pads of six-week-old female NCR nude mice (Charles River, Wilmington, MA, USA). A slow release pellet of $17 \beta$-estradiol (1.5 mg, 60-day release; Innovative Research of America, Toledo, $\mathrm{OH}, \mathrm{USA}$ ) was implanted subcutaneously in the dorsal interscapular region before cell transplantation. A total of 10 mice per cell line were used. Mice were monitored tri-weekly for the development of tumors, and necropsied after a three-week observation period. The tumor growth was monitored by measurements of tumor length $(\mathrm{L})$ and width $(\mathrm{W})$ and tumor volume was calculated as described. ${ }^{30}$ All animal studies were performed in compliance with the University of Southern California Institutional Animal Care and Use Committee (IACUC) guidelines.

Electron microscopy. Conventional electron microscopy was performed as described previously. ${ }^{31}$ Xenograft tumors were collected and fixed in Karnovsky's fixative overnight, postfixed with $2 \% \mathrm{OsO}_{4}$ for $1 \mathrm{~h}$, dehydrated with ethanol, and embedded in Epon-Araldite resin (Ted Pella Inc., Redding, CA, USA). $70 \AA$ ultrathin 
sections were cut with a Sorvall MT-6000 ultramicrotome. Before visualization, samples were stained in $1 \%$ aqueous uranyl acetate for $20 \mathrm{~min}$ and examined with a JEOL 2100 transmission electron microscope.

Histophathological and immunohistochemical analyses. Tumor sections from the indicated xenograft tumors were fixed in $10 \%$ buffered formalin, embedded in paraffin, and routinely stained with hematoxylin and eosin. Alternatively, the paraffin-embedded tumor sections were immunostained with the HA (1:1000; Covance, Princeton, NJ, USA), cleaved caspase-9 and PARP (1:50; Cell Signaling Technology, Danvers, MA, USA), $\gamma$-H2AX (1:50; Cell Signaling Technology), GRP78 (1:1000; Cell Signaling Technology), or p62 (1:50; Abcam, Cambridge, MA, USA) antibodies by standard procedure. The mitotic index was quantitated by viewing and photographing 10 random high-power-fields of each tissue section on a Nikon microscope, using a $\times 40$ objective.

Statistical analysis. Statistical analyses were performed using unpaired $t$-tests. Values are expressed as mean \pm S.D. of at least three independent experiments unless otherwise noted. A $P$ value of $\leq 0.05$ was considered statistically significant.

\section{Conflict of interest}

The authors declare no conflict of interest.

Acknowledgements. This work was partly supported by U.S. Public Health Service Grants CA140964, Al083841, the Leukemia \& Lymphoma Society of USA, the Wright Foundation, and the Baxter Foundation (C Liang), and CA82057, CA91819, CA31363, CA115284, Al073099, Fletcher Jones Foundation, Hastings Foundation, and Korean GRL Program K20815000001 (JUJ). X E has a Ruth L Krischstein National Research Service Award. We thank Drs. B Levine, MJ Hardwick, S Virgin, S Field, T Yoshimori, and $Y$ Ohsumi for providing reagents, $M$ Connole for FACS analysis, $P$ Furcinitti for technical help in imaging, $D$ Hauser and $E$ Barron for performing electron microscopy, and $L$ Young for immunohistochemistry analysis. Finally, we thank all the lab members for their support and discussions.

1. Yip KW, Reed JC. Bcl-2 family proteins and cancer. Oncogene 2008; 27: 6398-6406.

2. Youle RJ, Strasser A. The BCL-2 protein family: opposing activities that mediate cell death. Nat Rev Mol Cell Biol 2008; 9: 47-59.

3. Walensky LD. BCL-2 in the crosshairs: tipping the balance of life and death. Cell Death Differ 2006; 13: 1339-1350.

4. Petros AM, Olejniczak ET, Fesik SW. Structural biology of the Bcl-2 family of proteins. Biochim Biophys Acta 2004; 1644: 83-94.

5. Maiuri MC, Le Toumelin G, Criollo A, Rain JC, Gautier F, Juin P et al. Functional and physical interaction between $\mathrm{Bcl}-\mathrm{X}(\mathrm{L})$ and a BH3-like domain in Beclin-1. EMBO J 2007; 26: 2527-2539.

6. Pattingre S, Tassa A, Qu X, Garuti R, Liang XH, Mizushima N et al. Bcl-2 antiapoptotic proteins inhibit Beclin 1-dependent autophagy. Cell 2005; 122: 927-939.

7. Levine B, Sinha S, Kroemer G. Bcl-2 family members: dual regulators of apoptosis and autophagy. Autophagy 2008; 4: 600-606.

8. Levine B, Kroemer G. Autophagy in the pathogenesis of disease. Cell 2008; 132: 27-42.

9. Mizushima N, Levine B, Cuervo AM, Klionsky DJ. Autophagy fights disease through cellular self-digestion. Nature 2008; 451: 1069-1075.
10. Kihara A, Noda T, Ishihara N, Ohsumi Y. Two distinct Vps34 phosphatidylinositol 3-kinase complexes function in autophagy and carboxypeptidase $\mathrm{Y}$ sorting in Saccharomyces cerevisiae. J Cell Biol 2001; 152: 519-530.

11. Liang XH, Jackson S, Seaman M, Brown K, Kempkes B, Hibshoosh $\mathrm{H}$ et al. Induction of autophagy and inhibition of tumorigenesis by beclin 1. Nature 1999; 402: 672-676.

12. Mathew R, Kongara S, Beaudoin B, Karp CM, Bray K, Degenhardt K et al. Autophagy suppresses tumor progression by limiting chromosomal instability. Genes Dev 2007; 21: 1367-1381.

13. Karantza-Wadsworth V, Patel S, Kravchuk O, Chen G, Mathew R, Jin S et al. Autophagy mitigates metabolic stress and genome damage in mammary tumorigenesis. Genes Dev 2007; 21: 1621-1635.

14. Mathew R, Karp CM, Beaudoin B, Vuong N, Chen G, Chen HY et al. Autophagy suppresses tumorigenesis through elimination of p62. Cell 2009; 137: 1062-1075.

15. Akar U, Chaves-Reyez A, Barria M, Tari A, Sanguino A, Kondo Y et al. Silencing of Bcl-2 expression by small interfering RNA induces autophagic cell death in MCF-7 breast cancer cells. Autophagy 2008; 4: 669-679.

16. Xiaofei E, Hwang S, Oh S, Lee JS, Jeong JH, Gwack Y et al. Viral Bcl-2-mediated evasion of autophagy aids chronic infection of gammaherpesvirus 68. PLoS Pathog 2009; 5 : e1000609.

17. Oberstein A, Jeffrey PD, Shi Y. Crystal structure of the Bcl-XL-Beclin 1 peptide complex Beclin 1 is a novel BH3-only protein. J Biol Chem 2007; 282: 13123-13132.

18. Hirotani M, Zhang Y, Fujita N, Naito M, Tsuruo T. NH2-terminal BH4 domain of Bcl-2 is functional for heterodimerization with Bax and inhibition of apoptosis. J Biol Chem 1999; 274: 20415-20420.

19. Kabeya Y, Mizushima N, Ueno T, Yamamoto A, Kirisako T, Noda T et al. LC3, a mammalian homologue of yeast Apg8p, is localized in autophagosome membranes after processing. Embo J 2000; 19: 5720-5728.

20. Mizushima N, Yamamoto A, Matsui M, Yoshimori T, Ohsumi Y. In vivo analysis of autophagy in response to nutrient starvation using transgenic mice expressing a fluorescent autophagosome marker. Mol Biol Cell 2004; 15: 1101-1111.

21. Tanida I, Minematsu-lkeguchi N, Ueno T, Kominami E. Lysosomal turnover, but not a cellular level, of endogenous LC3 is a marker for autophagy. Autophagy 2005; 1: 84-91.

22. Mizushima N, Yoshimori T. How to interpret LC3 immunoblotting. Autophagy 2007; 3 542-545.

23. Bjorkoy G, Lamark T, Brech A, Outzen H, Perander M, Overvatn A et al. p62/SQSTM1 forms protein aggregates degraded by autophagy and has a protective effect on huntingtin-induced cell death. J Cell Biol 2005; 171: 603-614.

24. Qu X, Yu J, Bhagat G, Furuya N, Hibshoosh H, Troxel A et al. Promotion of tumorigenesis by heterozygous disruption of the beclin 1 autophagy gene. J Clin Invest 2003; 112: 1809-1820.

25. Abedin MJ, Wang D, McDonnell MA, Lehmann U, Kelekar A. Autophagy delays apoptotic death in breast cancer cells following DNA damage. Cell Death Differ 2007; 14: 500-510.

26. Reed JC, Kitada S, Takayama S, Miyashita T. Regulation of chemoresistance by the bcl-2 oncoprotein in non-Hodgkin's lymphoma and lymphocytic leukemia cell lines. Ann Oncol 1994; 5 (Suppl 1): 61-65.

27. Lee JS, Li Q, Lee JY, Lee SH, Jeong JH, Lee HR et al. FLIP-mediated autophagy regulation in cell death control. Nat Cell Biol 2009; 11: 1355-1362.

28. Cory S, Huang DC, Adams JM. The Bcl-2 family: roles in cell survival and oncogenesis. Oncogene 2003; 22: 8590-8607.

29. Liang C, Feng $\mathrm{P}, \mathrm{Ku}$ B, Dotan I, Canaani D, Oh BH et al. Autophagic and tumour suppressor activity of a novel Beclin1-binding protein UVRAG. Nat Cell Biol 2006; 8: 688-699.

30. Streit M, Velasco P, Brown LF, Skobe M, Richard L, Riccardi L et al. Overexpression of thrombospondin-1 decreases angiogenesis and inhibits the growth of human cutaneous squamous cell carcinomas. Am J Pathol 1999; 155: 441-452.

31. Yoshimori T, Yamagata F, Yamamoto A, Mizushima N, Kabeya Y, Nara A et al. The mouse SKD1, a homologue of yeast Vps4p, is required for normal endosomal trafficking and morphology in mammalian cells. Mol Biol Cell 2000; 11: 747-763.

32. Loh J, Huang $Q$, Petros AM, Nettesheim D, van Dyk LF, Labrada L et al. A surface groove essential for viral $\mathrm{Bcl}-2$ function during chronic infection in vivo. PLoS Pathog 2005; 1: e10.

Supplementary Information accompanies the paper on Cell Death and Differentiation website (http://www.nature.com/cdd) 